\title{
Matrix metalloproteinase haplotypes associated with coronary artery aneurysm formation in patients with Kawasaki disease
}

\author{
Chisato Shimizu ${ }^{1}$, Tomoyo Matsubara ${ }^{2}$, Yoshihiro Onouchi ${ }^{3}$, Sonia Jain ${ }^{4}$, Shelly Sun ${ }^{4}$, Caroline M Nievergelt ${ }^{5}$, \\ Hiroko Shike ${ }^{1}$, Victoria H Brophy ${ }^{6}$, Tsuyoshi Takegawa ${ }^{7}$, Susumu Furukawa ${ }^{7}$, Teiji Akagi ${ }^{8}$, Jane W Newburger ${ }^{9}$, \\ Annette L Baker ${ }^{9}$, David Burgner ${ }^{10,11}$, Martin L Hibberd ${ }^{12}$, Sonia Davila ${ }^{12}$, Michael Levin ${ }^{13}$, Manju Mamtani ${ }^{14}$, \\ Weijing $\mathrm{He}^{14}$, Sunil K Ahuja ${ }^{14}$ and Jane C Burns ${ }^{1}$
}

Aneurysms of the vascular wall represent a final common pathway for a number of inflammatory processes, including atherosclerosis and idiopathic vasculitis syndromes. Kawasaki disease (KD) is an acute, self-limited vasculitis in children and the leading cause of acquired coronary artery aneurysms. We sought to identify shared molecular mechanisms of aneurysm formation by genotyping eight polymorphisms in matrix metalloproteinase (MMP)-1, 3, 7, 12 and 13 in the gene cluster on Chr.11q22, whose gene products have been implicated in aneurysm formation or are known to have elastase activity. We genotyped 482 US-UK KD patients (aneurysm+: $n=111$, aneurysm-: $n=371$ ) and tested our findings in an independent cohort of 200 Japanese KD patients (aneurysm+: $n=58$, aneurysm-: $n=142$ ). Analysis of the five MMP genes identified modest trends in allele and genotype frequencies for MMP-3 rs3025058 (-/T) and haplotypes containing MMP-3 rs3025058 ( $-/ \mathrm{T})$ and MMP-12 rs2276109 (A/G) (nominal $P=2$ to $4 \times 10^{-5}$ ) that conferred increased risk of aneurysm formation in US-UK subjects. This finding was validated in Japanese subjects and suggests the importance of this locus in aneurysm formation in children with KD. The region encompassing these risk haplotypes is a prime candidate for resequencing to look for rare genetic variation that may influence aneurysm formation.

Journal of Human Genetics (2010) 55, 779-784; doi:10.1038/jhg.2010.109; published online 9 September 2010

Keywords: coronary artery aneurysm; haplotype; Kawasaki disease; matrix metalloproteinase

\section{INTRODUCTION}

Aneurysms of the vascular wall complicate many different diseases that involve vessel wall inflammation and destruction of extracellular matrix and elastic fibers. In children with Kawasaki disease (KD), coronary artery aneurysms (CAA) form in $25 \%$ of untreated patients and in $5 \%$ of patients treated with intravenous immunoglobulin within the first 10 days after fever onset. A hallmark of CAA is focal destruction of the internal elastic lamina with early neutrophil infiltration followed by macrophages and cytotoxic T lymphocytes. ${ }^{1}$ For this reason, enzymes that cleave elastin have been implicated in the pathogenesis of KD. ${ }^{2,3}$ Proteases capable of degrading elastin include neutrophil elastase and the matrix metalloproteinases (MMPs)-2, 3, 7, 9 and 12. ${ }^{4}$ MMPs are zinc-dependent endopeptidases produced by a wide variety of cell types. In addition to the degradation of extracellular matrix, MMPs also cleave cytokines and chemokines ${ }^{5}$ and influence recruitment of inflammatory cells. Therefore, MMPs have important roles in both inflammation and tissue remodeling.

According to a current paradigm, $\mathrm{KD}$ is triggered by an infectious agent that elicits an inflammatory response directed at cardiovascular tissues in genetically susceptible hosts. ${ }^{6}$ A genetic influence on disease susceptibility in KD has been explored in candidate gene association studies, a genome-wide linkage analysis of siblings concordant for KD followed by linkage disequilibrium mapping, and a genome-wide association study. ${ }^{7-11}$ However, fewer studies have explored the impact of genetic variation on aneurysm formation because of the difficulty in collecting a sufficient sample size of patients with this phenotype for genotyping. To bridge this gap in knowledge, we collaborated with groups in the United Kingdom and Japan to collect DNA from KD

\footnotetext{
${ }^{1}$ Rady Children's Hospital and Departments of Pediatrics, UCSD School of Medicine, La Jolla, CA, USA; ${ }^{2}$ Department of Pediatrics, Juntendo University Graduate School of Medicine, Urayasu Hospital, Chiba, Japan; ${ }^{3}$ Laboratory for Cardiovascular Diseases, Center for Genomic Medicine, RIKEN, Kanagawa, Japan; ${ }^{4}$ Department of Family and Preventive Medicine, UCSD School of Medicine, La Jolla, CA, USA; ${ }^{5}$ Department of Psychiatry, UCSD School of Medicine, La Jolla, CA, USA; ${ }^{6}$ Roche Molecular Systems Inc., Pleasanton, CA, USA; ${ }^{7}$ Department of Pediatrics, Yamaguchi University Graduate School of Medicine, Yamaguchi, Japan; ${ }^{8}$ Pediatrics Cardiac Care Unit, Okayama University Hospital, Okayama, Japan; ${ }^{9}$ Department of Cardiology, Boston Children's Hospital, Boston, MA, USA; ${ }^{10}$ School of Pediatrics and Child Health, University of Western Australia, Perth, Australia; ${ }^{11}$ Murdoch Childrens Research Institute, The Royal Childrens Hospital, Parkville, Australia; ${ }^{12}$ Infectious Diseases, Genome Institute of Singapore, Genome, Singapore; ${ }^{13}$ Imperial College, London, UK and ${ }^{14}$ University of Texas Health Science Center, San Antonio, TX, USA

Correspondence: Professor JC Burns, Department of Pediatrics-0641, UCSD School of Medicine, 9500 Gilman Drive, La Jolla, CA 92093-0641, USA.

E-mail: jcburns@ucsd.edu
}

Received 9 April 2010; revised 28 July 2010; accepted 29 July 2010; published online 9 September 2010 
patients and determined the contribution of five MMP genes (MMP1, 3, 7, 12 and 13) to CAA formation, as these MMPs have been implicated in atherosclerotic coronary artery (CA) and abdominal aneurysms or are known to have elastase activity. ${ }^{4,12,13}$ We genotyped eight single-nucleotide polymorphisms (SNPs) in these five MMP genes clustered on chromosome 11 and analyzed haplotypes in the US-UK cohort. These results were tested in an independent cohort of Japanese KD subjects to examine the association of this region with CAA across different ethnic populations.

\section{MATERIALS AND METHODS}

\section{Subjects}

Details of the US-UK subjects and their clinical presentation and case definition have been previously described. ${ }^{9}$ Briefly, KD patients $(n=482)$ were recruited at Rady Children's Hospital San Diego, CA; Boston Children's Hospital, Boston, MA; and Imperial College School of Medicine, London, UK. KD patients from Japan $(n=200)$ were recruited by investigators at Yamaguchi University and Oita Children's Hospital Japan. Parental consent and subject assent when appropriate were obtained for all subjects. The Institutional Review Boards of the participating centers reviewed and approved this study.

Demographic and clinical data were collected on all subjects as previously described $^{9}$ (Table 1). The distribution of different ethnic groups was similar between the CAA+ and CAA- cohorts. For the US subjects, CA status was assessed by echocardiography during the acute, subacute and convalescent phase of the illness. Measurements of the internal diameters of the proximal

Table 1 Characteristics of subjects with KD

\begin{tabular}{|c|c|c|c|c|}
\hline & \multicolumn{2}{|c|}{$U S-U K K D(\mathrm{n}=482)$} & \multicolumn{2}{|c|}{ Japanese $K D(\mathrm{n}=200)$} \\
\hline & $\begin{array}{c}C A A- \\
(\mathrm{n}=371)\end{array}$ & $\begin{array}{c}C A A+ \\
(\mathrm{n}=111)\end{array}$ & $\begin{array}{c}C A A- \\
(\mathrm{n}=142)\end{array}$ & $\begin{array}{c}C A A+ \\
(\mathrm{n}=58)\end{array}$ \\
\hline Male & $235(63 \%)$ & 77 (69\%) & $75(53 \%)$ & $40(69 \%)$ \\
\hline \multicolumn{5}{|l|}{ Self-reported ethnicity } \\
\hline Caucasian & $215(58 \%)$ & $75(68 \%)$ & & \\
\hline Caucasian-Hispanic & $56(15 \%)$ & $12(11 \%)$ & & \\
\hline Asian, unspecified & $42(11 \%)$ & $13(12 \%)$ & & \\
\hline Mixed & $43(12 \%)$ & $8(7 \%)$ & & \\
\hline Others & $14(4 \%)$ & $3(2 \%)$ & & \\
\hline Japanese & $1(0.2 \%)$ & $0(0 \%)$ & $142(100 \%)$ & $58(100 \%)$ \\
\hline
\end{tabular}

Abbreviations: CAA, coronary artery aneurysms; KD, Kawasaki disease. right (R) and left anterior descending CAs were normalized for body surface area and expressed as standard deviation units from the mean ( $Z$-scores). For US subjects, CAA was defined as a $Z$-score $\geqslant 4.0$ in the first year after $\mathrm{KD}$ onset in association with an internal diameter $\geqslant 1.5$ times the adjacent segment. ${ }^{14}$ For the UK and Japanese subjects, $Z$-scores were not available and CA lesions (aneurysm or ectasia) were defined according to the Japanese Ministry of Health criteria (internal lumen diameter $\geqslant 3 \mathrm{~mm}$ for children $<5$ years and $\geqslant 4 \mathrm{~mm}$ for children $\geqslant 5$ years or the internal diameter of one or more segments $\geqslant 1.5$ times the diameter of the adjacent segment. ${ }^{15}$

\section{Genotyping}

Genomic DNA from whole blood or mouth wash samples was extracted as previously described. ${ }^{9}$ We chose eight SNPs from five MMP genes (Figure 1) that had positive disease associations in the published literature ${ }^{16-23}$ (Figure 2). Genotyping was performed using a multiplex PCR-based, sequence-specific oligonucleotide hybridization research assay (Roche Molecular Systems, Pleasanton, CA, USA) as previously described. ${ }^{9}$ To genotype MMP13 rs2252070 (A/G), we used a TaqMan allele discrimination assay (Applied Biosystem, Foster City, CA, USA, Assay ID: C_25474083_10) according to the manufacturer's instructions. Different numbers of subjects were genotyped for different loci because of limited availability of some of the genotyping reagents that were

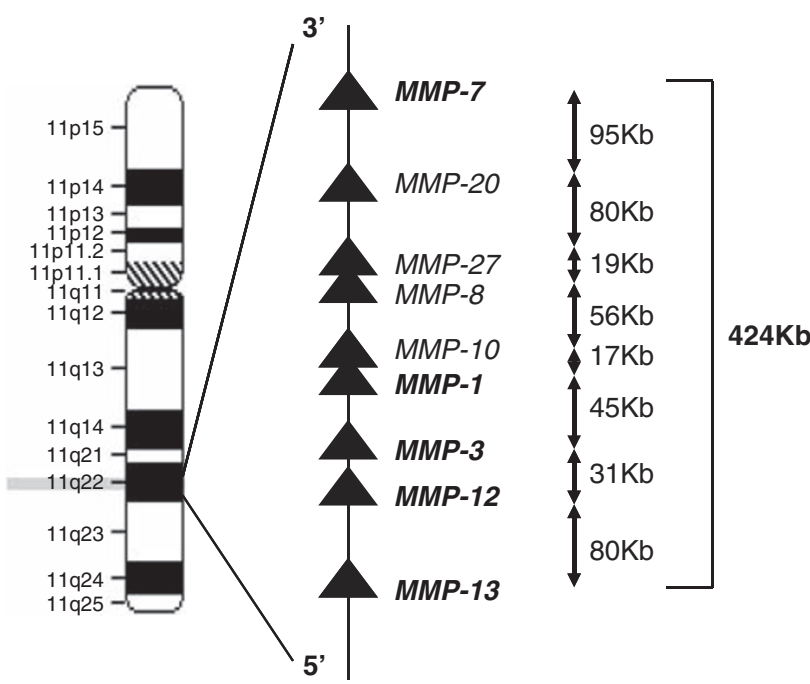

Figure 1 Matrix metalloproteinase (MMP) gene cluster on $11 \mathrm{q} 22$. Arrowheads show the direction of genes. Arrows on the right indicate intergenic distance in the $424 \mathrm{~Kb}$ region. Genes in bold were genotyped in this study.

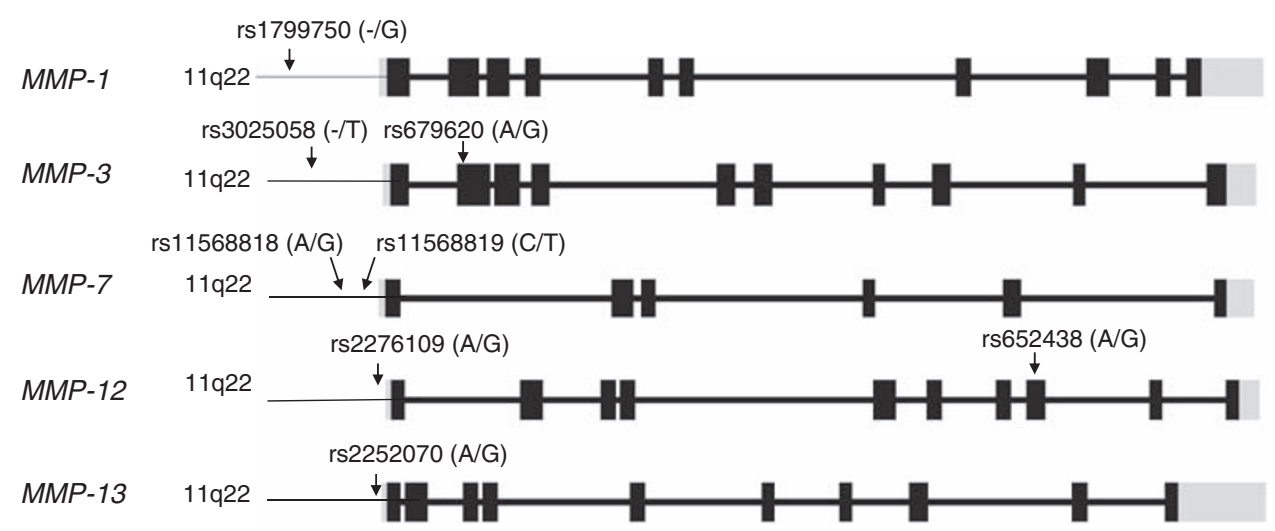

Figure 2 Eight single-nucleotide polymorphisms (SNPs) from five matrix metalloproteinase (MMP) genes genotyped in Kawasaki disease patients. Gene structure and the location of SNPs: black boxes, exons; gray boxes, $3^{\prime}$ and $5^{\prime}$ untranslated regions. 
discontinued by the manufacturer before the study was completed (Supplementary Tables 1 and 2). Data quality control was performed as previously described. ${ }^{9}$ SNPs with allele frequencies $<1 \%$, genotype call rate $<93 \%$ or deviation from Hardy-Weinberg equilibrium were excluded from further analysis.

\section{Statistical analyses}

Case-control association studies between MMP SNPs and CA status were analyzed by the general linear model: $Y_{i}=\alpha+\beta X_{i}+\epsilon_{i}$. The genetic models that were considered were the codominant model (genotype) and the additive model (allele). Under the codominant model, a particular SNP is considered a categorical variable with one level for each genotype for a total of three levels. To assess the association between phenotype $\mathrm{Y}$ and each SNP, the above general linear model is used, where $\alpha$ represents the intercept, $X_{i}$ is the $i$ th subject's genotype score for a given marker and $\epsilon_{i}$ is normally distributed with mean 0 and variance $\sigma^{2}$. Under the dominant model, $X_{i}=1$ if the $i$ th subject has at least one minor allele or $X_{i}=0$ otherwise. Under the additive model, $X_{i}$ indicates the $i$ th subject's number of minor alleles. This is equivalent to the test based on the allele frequency. Multiple testing corrections were not applied and nominal $P$-values are shown in tables. Analysis was performed using the $\mathrm{R}$ software (version 2.6.2, http://www.r-project.org/) and the R package SNPassoc.

Haplotype associations between MMP SNPs and CA status were analyzed using a moving window approach. The expectation-maximization algorithm ${ }^{24}$ was used to estimate haplotype frequencies and to account for missing genotypes. Score statistics were computed to test associations between the haplotypes and various traits. Analyses were performed using the $\mathrm{R}$ package haplo.stats. ${ }^{25}$ Correction for multiple testing was performed for single locus and haplotype analyses according to the following calculation: single locus: 0.05/number of SNPs tested; haplotypes: 0.05/number of observed haplotypes/ number of haplotypes with nominal $P<0.05$.

\section{RESULTS}

Single-locus analysis in the US-UK cohort

Case-control analysis of the multiethnic US-UK cohort (111 KD subjects with aneurysms vs $371 \mathrm{KD}$ subjects with normal or transiently dilated CAs) was performed and genotype data met quality control criteria on eight SNPs in five MMP genes. Modest trends in allele and genotype frequencies were noted between the two groups for $M M P-3$ rs3025058 $(-/ \mathrm{T})$ (allele nominal $P=0.031$, odds ratio $=0.72,95 \%$ confidence interval 0.54-0.97; genotype nominal $P=0.015$ ), suggesting that the $\mathrm{T}$ allele in the $M M P-3$ promoter contributes to protection against aneurysm formation. Another SNP in MMP-3, rs679620 (A/G) was associated with a trend towards protection (allele nominal $P=0.089$, odds ratio $=0.76,95 \%$ confidence interval $0.56-1.05$; genotype nominal $P=0.042$ ) (Supplementary Table 1). None of these associations remained significant after correction for multiple testing. To explore the possible effect of population stratification on our analysis, we performed single-locus analyses using only self-reported Caucasian subjects (CAA $+n=75, \mathrm{CAA}-n=215$ ). No significant differences between the CAA+ and CAA- groups were detected with this smaller sample (data not shown).

\section{Haplotype analysis in the US-UK subjects}

We analyzed SNPs in the five MMP genes located in a cluster on chromosome 11q22 (MMP-1, 3, 7, 12 and 13) (Figure 1). Several haplotypes were associated with CAA formation in the US-UK subjects (Table 2). Analysis using a window of two SNPs identified two haplotypes both with the del allele (-allele) of MMP-3 rs3025058 $(-/ \mathrm{T})$ that were associated with increased risk of aneurysm formation (nominal $P=0.03$ and 0.04 , respectively). The association of the del allele of MMP-3 rs3025058 (-/T) with the A allele of rs679620 (A/G) and the A allele of rs2276109 (A/G) in MMP-12 remained constant, suggesting a strong linkage disequilibrium in this region $\left(\mathrm{D}^{\prime}\right.$ between MMP-3 rs3025058 (-/T) and rs679620 (A/G): 0.92, MMP-3 rs3025058 (-/T) and MMP-12 rs2276109 (A/G): 0.85). Haplotypes containing three to eight SNPs that were associated with CAA all included the del allele of $M M P-3$ rs3025058 (-/T). Haplotypes

Table 2 Haplotypes on Chr. 11q22 associated with coronary artery aneurysms in the multiethnic US-UK cohort

\begin{tabular}{|c|c|c|c|c|c|c|c|c|c|c|c|}
\hline \multicolumn{8}{|c|}{ Haplotype } & \multirow{3}{*}{$\begin{array}{c}\text { Total number } \\
\text { of observed } \\
\text { haplotypes }\end{array}$} & \multirow{2}{*}{\multicolumn{2}{|c|}{ Frequency }} & \multirow[b]{3}{*}{ P-value } \\
\hline \multicolumn{2}{|c|}{$M M P-7$} & \multirow{2}{*}{$\begin{array}{c}M M P-1 \\
\text { rs1 } 799750-/ G\end{array}$} & \multicolumn{2}{|c|}{$M M P-3$} & \multicolumn{2}{|c|}{ MMP-12 } & \multirow{2}{*}{$\begin{array}{c}M M P-13 \\
r s 2252070 A / G\end{array}$} & & & & \\
\hline$r s 11568819 \mathrm{C} / \mathrm{T}$ & rs11568818A/G & & $r s 679620 A / G$ & rs3025058-/T & rs652438A/G & $r s 2276109 A / G$ & & & $C A A-$ & $C A A+$ & \\
\hline & & & A & - & & & & 4 & 0.4 & 0.49 & 0.03 \\
\hline & & & & - & $A$ & & & 4 & 0.4 & 0.48 & 0.04 \\
\hline & & & A & - & A & & & 7 & 0.4 & 0.48 & 0.04 \\
\hline & & & & - & A & A & & 7 & 0.4 & 0.47 & 0.05 \\
\hline & & & & - & A & A & $\mathrm{G}$ & 11 & 0.05 & 0.12 & 0.002 \\
\hline & & & $A$ & - & $A$ & $A$ & $G$ & 16 & 0.05 & 0.12 & 0.004 \\
\hline & & - & $A$ & - & $A$ & $A$ & $\mathrm{G}$ & 27 & 0.05 & 0.08 & 0.03 \\
\hline & $A$ & - & $A$ & - & $A$ & $A$ & G & 37 & 0.01 & 0.08 & 0.001 \\
\hline C & $A$ & - & A & - & $A$ & $A$ & $\mathrm{G}$ & 46 & 0.01 & 0.08 & 0.001 \\
\hline C & $A$ & G & A & - & & & & 18 & 0.03 & 0.07 & 0.03 \\
\hline C & $A$ & $\mathrm{G}$ & $A$ & - & $A$ & & & 24 & 0.03 & 0.07 & 0.02 \\
\hline \multirow[t]{5}{*}{ C } & A & $G$ & $A$ & - & $A$ & $A$ & & 30 & 0.03 & 0.06 & 0.02 \\
\hline & $A$ & G & $A$ & - & $A$ & A & & 25 & 0.03 & 0.06 & 0.04 \\
\hline & A & G & A & - & $A$ & & & 19 & 0.03 & 0.06 & 0.05 \\
\hline & & G & A & - & A & A & G & 27 & 0.01 & 0.04 & 0.001 \\
\hline & A & G & A & - & A & A & G & 37 & 0.005 & 0.04 & $0.00002^{b}$ \\
\hline C & $A$ & $G$ & $A$ & - & $A$ & $A$ & $\mathrm{G}$ & 46 & 0.004 & 0.05 & $0.00004^{c}$ \\
\hline
\end{tabular}

Abbreviations: CAA, coronary artery aneurysms; KD, Kawasaki disease; MMP, matrix metalloproteinase.

Threshold for significance after correction for multiple testing: $\mathrm{b}=8 \times 10^{-5} ; \mathrm{c}=6 \times 10^{-5}$. KD subjects with normal or dilated coronary arteries (CAA-, $n=371$ ) were compared with $\mathrm{KD}$ subjects with aneurysms (CAA+, $n=111)$

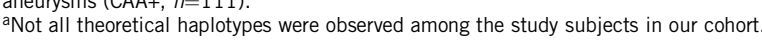


Table 3 Haplotypes on Chr. 11q22 associated with coronary artery aneurysms in the Caucasian-only US-UK cohort

\begin{tabular}{|c|c|c|c|c|c|c|c|c|c|c|c|}
\hline \multicolumn{8}{|c|}{ Haplotype } & \multirow{3}{*}{$\begin{array}{l}\text { Total number } \\
\text { of observed } \\
\text { haplotypes }\end{array}$} & \multirow{2}{*}{\multicolumn{2}{|c|}{ Frequency }} & \multirow[b]{3}{*}{$\mathrm{P}$-value } \\
\hline \multicolumn{2}{|c|}{$M M P-7$} & \multirow{2}{*}{$\begin{array}{c}M M P-1 \\
\text { rs1799750 -/G }\end{array}$} & \multicolumn{2}{|c|}{$M M P-3$} & \multicolumn{2}{|c|}{$M M P-12$} & \multirow{2}{*}{$\begin{array}{c}M M P-13 \\
r s 2252070 \mathrm{~A} / \mathrm{G}\end{array}$} & & & & \\
\hline rs11568819 C/T & rs11568818 A/G & & $r s 679620 \mathrm{~A} / \mathrm{G}$ & rs3025058 - /T & $r s 652438 A / G$ & rs2276109 A/G & & & CAA- & $C A A+$ & \\
\hline & $A$ & $G$ & A & - & $A$ & $A$ & $G$ & 30 & 0.01 & 0.10 & $0.0034^{b}$ \\
\hline C & A & G & A & - & $A$ & A & G & 36 & 0.02 & 0.09 & $0.0039^{b}$ \\
\hline & $A$ & - & G & $\mathrm{T}$ & $A$ & & & 17 & 0.12 & 0.05 & 0.041 \\
\hline C & $A$ & - & G & $\mathrm{T}$ & $A$ & & & 18 & 0.11 & 0.05 & 0.047 \\
\hline & $A$ & - & G & $\mathrm{T}$ & $A$ & $A$ & & 22 & 0.12 & 0.05 & 0.039 \\
\hline C & $A$ & - & $G$ & $\mathrm{~T}$ & $A$ & $A$ & & 23 & 0.11 & 0.05 & 0.041 \\
\hline
\end{tabular}

Abbreviations: CAA, coronary artery aneurysms; KD, Kawasaki disease; MMP, matrix metalloproteinase.

KD subjects with normal or dilated coronary arteries (CAA-, $n=215)$ were compared with KD subjects with aneurysms (CAA+, $n=75)$.

aNot all theoretical haplotypes were observed among the study subjects in our cohort.

bHaplotype also associated with CAA formation in the multiethnic cohort (Table 2).

Table 4 Haplotypes on Chr. 11q22 associated with CAA in Japanese subjects

\begin{tabular}{|c|c|c|c|c|c|c|c|}
\hline \multicolumn{4}{|c|}{ Haplotypes } & \multirow[b]{3}{*}{ Total number of haplotypes } & \multirow{2}{*}{\multicolumn{2}{|c|}{ Frequency }} & \multirow[b]{3}{*}{ P-value } \\
\hline \multicolumn{2}{|c|}{$M M P-3$} & \multicolumn{2}{|c|}{$M M P-12$} & & & & \\
\hline rs679620 A/G & rs3025058 -/T & rs652438 A/G & $r s 2276109 \mathrm{~A} / \mathrm{G}$ & & $\mathrm{CAA}-$ & $C A A+$ & \\
\hline & & $A$ & $\mathrm{G}$ & 3 & 0.03 & 0.11 & $0.003^{b}$ \\
\hline & $\mathrm{T}$ & $A$ & G & 6 & 0.01 & 0.10 & $0.0009^{c}$ \\
\hline G & $\mathrm{T}$ & A & G & 8 & 0.01 & 0.10 & $0.001^{d}$ \\
\hline
\end{tabular}

Abbreviations: CAA, coronary artery aneurysms; KD, Kawasaki disease; MMP, matrix metalloproteinase.

KD subjects with CAA ( $n=58$ ) was compared with KD subjects without CAA ( $n=142)$. Threshold for significance after correction for multiple testing: $\mathrm{b}=0.006, \mathrm{c}=0.003, \mathrm{~d}=0.002$.

${ }^{a}$ Not all theoretical haplotypes were observed among the study subjects in our cohort.

containing the $\mathrm{T}$ allele were not significantly associated. Two haplotypes were significant after correction for multiple sample testing (Table 2, nominal $P=2$ to $4 \times 10^{-5}$ ). The risk haplotypes consistently included the $\mathrm{G}$ allele of $M M P-13 \mathrm{rs} 2252070(\mathrm{~A} / \mathrm{G})$, the $\mathrm{A}$ and $\mathrm{C}$ alleles of MMP7 rs11568818 (A/G) and rs11568819 (C/T), respectively. To explore the possible effects of population stratification on our analysis, we performed haplotype analyses using only self-reported Caucasian subjects from the larger, multiethnic cohort (CAA+ $n=75$, CAA$n=215$ ) (Table 3). Two haplotypes identified in the multiethnic cohort again showed association with CAA (nominal $P=0.0034$ and 0.0039 ) in the all-Caucasian subset. Four 5-7 SNP haplotypes were associated with protection against aneurysm formation, whereas these same haplotypes with the opposite alleles at MMP-1 rs1799750 (-/G), MMP-3 rs679620 (A/G) and rs3025058 (-/T) were associated with aneurysm formation in the larger, multiethnic cohort.

\section{Single-locus analysis in Japanese cohort}

To test the influence of genetic variation in MMP-3 and MMP-12 in a different ethnic cohort, single-locus analysis for CAA in the Japanese cohort was performed for SNPs in MMP-3 and MMP-12. MMP-12 rs2276109 (A/G) was associated with CAA formation with the $G$ allele conferring increased risk $(P=0.006$, odds ratio $=4.92,95 \%$ confidence interval 1.54-15.73) (Supplementary Table 2).

\section{Haplotype analysis in Japanese subjects}

We analyzed the haplotypes of MMP-3 and MMP-12 in a Japanese cohort and found that haplotypes containing the MMP-12 rs2276109 (A/G) G allele and MMP-3 rs3025058 (-/T) T allele were associated with CAA after correction for multiple testing (nominal $P=0.003-$ 0.0009); however, these haplotypes were rare in the study cohort (frequencies: CAA-: 0.01, CAA+: 0.10) (Table 4).

\section{DISCUSSION}

We tested the hypothesis that genetic variation in MMP genes influences CA damage in patients with KD. Haplotypes in $M M P-3$ and $M M P-12$, both with elastolytic activity, were associated with aneurysm formation in our mixed ethnic cohort from the US-UK and an all-Caucasian subset of the cohort. Analysis of an independent cohort of Japanese KD subjects validated the influence of haplotypes in $M M P-3$ and $M M P-12$ on aneurysm formation, although different alleles were associated with increased risk in the Japanese cohort. This may be due to a difference in haplotype structure with the consequence that the functional genetic variant is linked differently to these alleles in the two populations. Fine mapping or resequencing of this region in these two populations may uncover the specific genetic variation associated with CAA formation.

We found a relationship between haplotypes, including SNPs in MMP-12, and aneurysm formation in KD. MMP-12, which encodes for a macrophage elastase, has multiple functions that could be important in aneurysm pathogenesis. In addition to degrading extracellular matrix proteins, MMP-12 may promote macrophage recruitment to the vessel wall by activating tumor necrosis factor- $\alpha$ or by modulating levels of proinflammatory cytokines such as monocyte chemotactic protein-1. ${ }^{26}$ The A allele of $M M P-12 \mathrm{rs} 2276109(\mathrm{~A} / \mathrm{G})$ shows a higher affinity for the transcription factor activator protein-1 (AP-1) and higher expression levels in reporter gene assays. ${ }^{17} \mathrm{MMP}-12$ 
rs652438 (A/G) in exon 8 changes the neutral amino acid, asparagine, to the neutral amino acid, serine, in the hemopexin domain, which is thought to bind to TIMP-1. Functional studies have not been performed to determine the consequence of this amino-acid substitution.

The haplotypes associated with aneurysm formation also included MMP-3. MMP-3 also has elastolytic capabilities and has an important role in other diseases involving vascular wall inflammation, including AAA, atherosclerosis, and Takaysu's arteritis. ${ }^{27}$ MMP-3 rs3025058 $(-/ \mathrm{T})$ is a well-established functional variant. The $\mathrm{T}$ allele in this promoter region preferentially binds to the transcriptional repressor, nuclear factor- $\kappa \mathrm{B}$ p50 homodimer, and is associated with reduced transcript abundance. ${ }^{19}$ MMP-3 rs679620 (A/G) is located close to a cleavage site, and the substitution of positively charged lysine for negatively charged glutamic acid may alter protein function. In our US-UK subjects, haplotype analysis identified the del-A as the risk haplotype. Our findings are in agreement with a study of $M M P-3$ rs3025058 $(-/ \mathrm{T})$ in a small Korean cohort of KD subjects (CAA+, $n=34$; CAA-, $n=49) .{ }^{28}$ Homozygotes for the MMP-3 T allele had increased risk of CAA. Although this locus was associated with CAA in both the Japanese and US-UK subjects, different alleles were implicated suggesting a difference in linkage disequilibrium structure in this region. As for $M M P-12$, this region should be considered for resequencing efforts to further understand the role of genetic variation on CAA.

Recently, the A allele of MMP-13 rs2252070 (A/G) was reported to be associated with CAA formation in Japanese cohorts (CAA+, $n=44$; CAA,$- n=92) .{ }^{29} M M P-13$ rs 2252070 (A/G) was only genotyped in the US-UK cohort in our study and was not significantly associated in the single-locus analysis (CAA+, $n=62$; CAA,$- n=202$ ). The risk haplotype for CAA formation included the G allele of MMP-13 rs2252070 $(A / G)$ in our US-UK cohort, which differs from the published results in Japanese cohorts. Variation in population structure or our larger sample size may account for these differences. The effect of genetic variation in $M M P-13$ on CAA formation in Japanese subjects should be explored in future studies.

Discrepant results were obtained for the effect of MMP-1 rs 1799750 on CAA in different cohorts. In the multiethnic US-UK cohort, both alleles of MMP-1 rs1799750 (-/G) were in haplotypes associated with CAA (Table 2). However, in the Caucasian-only analysis (Table 3), the deletion allele MMP-1 $\mathrm{rs} 1799750$ (-/G) was associated with protection against CAA, whereas the $\mathrm{G}$ allele was associated with increased risk. One possible interpretation is that the linkage disequilibrium structure in Caucasians is different, thus driving the difference in results.

We recognize several limitations to our study. First, we performed case-control analyses using a multiethnic US-UK cohort to increase the statistical power. We justified this on the basis of similar allele frequencies in HapMap for the MMP SNPs in Caucasian-Hispanics and Caucasians, which comprised $85 \%$ of our cohort. Analyses performed on an all-Caucasian subset with reduced sample size supported the results from the analysis of the multiethnic cohort. Although this is one of the largest studies on genetic determinants influencing CAA formation, the number of subjects with CAA was still limited and results will need to be validated in additional, independent cohorts. Our study did not replicate the association of SNPs in MMP $13,{ }^{29}$ but a smaller cohort was genotyped for this region in our study, thus reducing our power to detect an association. This should be addressed in future studies with a larger sample size. As another limitation, our genotyping approach did not include all haplotype-tagging SNPs across the MMP gene cluster and not all genes/SNPs were genotyped in both cohorts. Detailed fine mapping or resequencing of $M M P-3$ and $M M P-12$ should be considered in future studies. Finally, the CA phenotyping varied slightly between the western countries and Japan. Some subjects in the Japanese aneurysm group might have been classified as transiently dilated according to the US criteria and therefore included with the normal group. However, the number of subjects who might have been differently assigned in the US and Japanese cohorts would have been small.

In summary, haplotype analyses suggested an influence of genetic variation in genes for the elastolytic MMPs in the gene cluster on Chr.11 on formation of CAA in KD patients, which was validated in an independent cohort. MMP-3 and MMP-12 haplotypes have been associated with susceptibility to aneurysms in other conditions, which suggests a shared molecular mechanism that unifies these inflammation-associated aneurysm syndromes.

\section{ACKNOWLEDGEMENTS}

We thank Tamotsu Fujimoto, MD (Pediatrics, Oita Children's Hospital), Joan Pancheri RN, Ellen McGrath RN, Jennifer Foley RN and Jon Goulding, PhD, for DNA collection and DeeAnna Scherrer and Clay Archer for laboratory assistance. We also thank Suzanne Cheng, PhD (Roche Molecular Systems), for helpful discussion. This work was supported in part by grants from the National Institutes of Health and the National Heart, Lung, Blood Institute (HL074864 and HL69413) awarded to JCB.

1 Takahashi, K., Oharaseki, T., Naoe, S., Wakayama, M. \& Yokouchi, Y. Neutrophilic involvement in the damage to coronary arteries in acute stage of Kawasaki disease. Pediatr Int. 47, 305-310 (2005).

2 Inamo, Y. Harada, K., Okuni, M., Kimoto, K., Takeuchi, S. \& Sakurabayashi, I. Immunoreactive polymorphonuclear leukocyte elastase in complex with alpha 1-antitrypsin in Kawasaki disease. Acta Paediatr Jpn. 29, 202-205 (1987).

3 Biezeveld, M. H., van Mierlo, G., Lutter, R., Kuipers, I. M., Dekker, T., Hack, C. E. et al. Sustained activation of neutrophils in the course of Kawasaki disease: an association with matrix metalloproteinases. Clin Exp Immunol. 141, 183-188 (2005).

4 Shapiro, S. D. Matrix metalloproteinase degradation of extracellular matrix: biological consequences. Curr Opin Cell Biol. 10, 602-608 (1998)

5 Manicone, A. M. \& McGuire, J. K. Matrix metalloproteinases as modulators of inflammation. Semin Cell Dev Biol. 19, 34-41 (2008).

6 Burns, J. C. \& Glode, M. P. Kawasaki syndrome. Lancet. 364, 533-544 (2004).

7 Burgner, D., Davila, S., Breunis, W. B., Ng, S. B., Li, Y., Bonnard, C. et al. A genomewide association study identifies novel and functionally related susceptibility Loci for Kawasaki disease. PLoS genetics. 5, e1000319 (2009).

8 Burns, J. C., Shimizu, C., Gonzalez, E., Kulkarni, H., Patel, S., Shike, H. et al. Genetic variations in the receptor-ligand pair CCR5 and CCL3L1 are important determinants of susceptibility to Kawasaki disease. J Infect Dis. 192, 344-349 (2005).

9 Burns, J. C., Shimizu, C., Shike, H., Newburger, J. W., Sundel, R. P., Baker, A. L. et al. Family-based association analysis implicates IL-4 in susceptibility to Kawasaki disease. Genes Immun. 6, 438-444 (2005).

10 Onouchi, Y., Gunji, T., Burns, J. C., Shimizu, C., Newburger, J. W., Yashiro, M. et al. ITPKC functional polymorphism associated with Kawasaki disease susceptibility and formation of coronary artery aneurysms. Nat Genet. 40, 35-42 (2008).

11 Onouchi, Y., Tamari, M., Takahashi, A., Tsunoda, T., Yashiro, M., Nakamura, Y. et al. A genomewide linkage analysis of Kawasaki disease: evidence for linkage to chromosome 12. J Hum Genet. 52, 179-190 (2007).

12 Aziz, F. \& Kuivaniemi, H. Role of matrix metalloproteinase inhibitors in preventing abdominal aortic aneurysm. Ann Vasc Surg. 21, 392-401 (2007).

13 Thompson, R. W. \& Parks, W. C. Role of matrix metalloproteinases in abdominal aortic aneurysms. Ann NY Acad Sci. 800, 157-174 (1996).

14 Newburger, J. W., Takahashi, M., Gerber, M. A., Gewitz, M. H., Tani, L. Y., Burns, J. C. et al. Diagnosis, treatment, and long-term management of Kawasaki disease: a statement for health professionals from the Committee on Rheumatic Fever, Endocarditis and Kawasaki Disease, Council on Cardiovascular Disease in the Young, American Heart Association. Circulation. 110, 2747-2771 (2004).

15 Disease, R.C.o.K. Report of subcommittee on standardization of diagnostic criteria and reporting of coronary artery lesions in Kawasaki disease (Ministry of Health and Welfare, Tokyo, Japan, 1984)

16 Jormsjo, S., Whatling, C., Walter, D. H. Zeiher, A. M., Hamsten, A. \& Eriksson, P. Allele-specific regulation of matrix metalloproteinase-7 promoter activity is associated with coronary artery luminal dimensions among hypercholesterolemic patients. Arterioscler Thromb Vasc Biol. 21, 1834-1839 (2001).

17 Jormsjo, S., Ye, S., Moritz, J., Walter, D. H., Dimmeler, S., Zeiher, A. M. et al. Allelespecific regulation of matrix metalloproteinase-12 gene activity is associated with 
coronary artery luminal dimensions in diabetic patients with manifest coronary artery disease. Circ Res. 86, 998-1003 (2000).

18 Rutter, J. L., Mitchell, T. I., Buttice, G., Meyers, J., Gusella, J. F., Ozelius, L. J. et al. A single nucleotide polymorphism in the matrix metalloproteinase-1 promoter creates an Ets binding site and augments transcription. Cancer Res. 58, 5321-5325 (1998).

19 Ye, S., Eriksson, P., Hamsten, A., Kurkinen, M., Humphries, S. E. \& Henney, A. M. Progression of coronary atherosclerosis is associated with a common genetic variant of the human stromelysin-1 promoter which results in reduced gene expression. $J$ Biol Chem. 271, 13055-13060 (1996).

20 Yoon, S., Kuivaniemi, H., Gatalica, Z., Olson, J. M., Buttice, G., Ye, S. et al. MMP13 promoter polymorphism is associated with atherosclerosis in the abdominal aorta of young black males. Matrix Biol. 21, 487-498 (2002).

21 Haq, I., Chappell, S., Johnson, S. R., Lotya, J., Daly, L., Morgan, K. et al. Association of MMP-2 polymorphisms with severe and very severe COPD: a case control study of MMPs-1, 9 and 12 in a European population. BMC Medical Genetics. 11, 7.

22 Joos, L., He, J. Q., Shepherdson, M. B., Connett, J. E., Anthonisen, N. R., Pare, P. D. et al. The role of matrix metalloproteinase polymorphisms in the rate of decline in lung function. Hum. Mol Genetics. 11, 569-576 (2002).
23 Ricketts, C., Zeegers, M. P., Lubinski, J. \& Maher, E. R. Analysis of germline variants in CDH1, IGFBP3, MMP1, MMP3, STK15 and VEGF in familial and sporadic renal cell carcinoma. PLoS One. 4, e6037 (2009).

24 Dempster, A., Laird, N. \& Rubin, D. Likelihood from incomplete data via the EM algorithm. J. Royal Stat. Soc. Series B. 39, 1-38 (1977).

25 Sinnwell, J. P. \& Schaid, D. J. haplo.stats: statistical analysis of haplotypes with traits and covariates when linkage phase is ambiguous $R$ package version 1.2.2., (2005).

26 Hautamaki, R. D., Kobayashi, D. K., Senior, R. M. \& Shapiro, S. D. Requirement for macrophage elastase for cigarette smoke-induced emphysema in mice. Science. 277, 2002-2004 (1997).

27 Matsuyama, A., Sakai, N., Ishigami, M., Hiraoka, H., Kashine, S., Hirata, A. et al. Matrix metalloproteinases as novel disease markers in Takayasu arteritis. Circulation. 108, 1469-1473 (2003).

28 Park, J. A., Shin, K. S. \& Kim, Y. W. Polymorphism of matrix metalloproteinase-3 promoter gene as a risk factor for coronary artery lesions in Kawasaki disease. $J$ Korean Med Sci. 20, 607-611 (2005).

29 Ikeda, K., Ihara, K., Yamaguchi, K., Muneuchi, J., Ohno, T., Mizuno, Y. et al. Genetic analysis of MMP gene polymorphisms in patients with Kawasaki disease. Pediatr Res. $63,182-185$ (2008).

Supplementary Information accompanies the paper on Journal of Human Genetics website (http://www.nature.com/jhg) 